\title{
Isoenzimas de adenosina deaminasa en líquido cefalorraquídeo como ayuda diagnóstica en tuberculosis meníngea
}

\section{Cerebrospinal fluid adenosine deaminase isoenzymes in meningeal tuberculosis diagnosis}

\author{
Helí Jaime Barrón-Pastor ${ }^{1,2}$, Ruth Isela Cisneros-Chinchay ${ }^{1,2,3}$, \\ Álvaro Julián Marcelo-Rodríguez ${ }^{2,4}$ \\ 1 Departamento Académico de Ciencias Dinámicas, Universidad Nacional Mayor de San Marcos, Lima, Perú. \\ ${ }^{2}$ Centro de Investigación de Bioquímica y Nutrición, Facultad de Medicina, Universidad Nacional Mayor de San Marcos, Lima, Perú. \\ ${ }^{3}$ Hospital Nacional Guillermo Almenara Irigoyen, EsSalud, Lima, Perú. \\ ${ }^{4}$ Facultad de Ciencias Biológicas, Universidad Nacional Mayor de San Marcos, Lima, Perú.
}

\begin{abstract}
Resumen
Introducción: La tuberculosis (TB) sigue siendo uno de las principales causas de muerte en el mundo. La TB meníngea como complicación requiere detección pronta e instalación inmediata de la terapia apropiada. Dada la baja sensibilidad de la baciloscopia y del cultivo del bacilo tuberculoso, se presenta el estudio de las isoenzimas adenosina deaminasa (ADA) como contribución al diagnóstico diferencial de la TB meníngea. Objetivo: Determinar la actividad de isoenzimas ADA en líquido cefalorraquideo (LCR) de pacientes con TB meníngea. Diseño: Investigación descriptiva, con muestreo no probabilístico. Institución: Centro de Investigación de Bioquímica y Nutrición, Universidad Nacional Mayor de San Marcos. Material biológico: Muestras de LCR de pacientes con TB. Intervenciones: Se consideró muestras de LCR con actividad ADA mayor a $9 \mathrm{U} / \mathrm{L}$, que fueron conservadas a $-40{ }^{\circ} \mathrm{C}$ hasta la corrida electroforética en sistema vertical. El análisis estadístico se realizó aplicando Mann - Withney, con un nivel de significancia de 0,1 para comparar los valores ADA de TB meníngea frente a otras enfermedades del sistema nervioso central (SNC). Principales medidas de resultados: Isoenzimas ADA1m, ADA1cp y ADA2. Resultados: Las medianas de la actividad de ADA total en LCR de pacientes tuberculosos fueron mayores que en otras enfermedades parainfectivas del SNC. ADA1cp tuvo mayor contribución para ADA total en ambos casos, mostrando mayor incremento en tuberculosis meníngea. También, ADA2 aumentó en TB meníngea frente a otras enfermedades del SNC. Conclusiones: Las isoenzimas ADA en LCR expresan niveles elevados de ADA2 en tuberculosis meníngea, como consecuencia del incremento de la línea celular monocito-macrófago.
\end{abstract}

Palabras clave: Tuberculosis meníngea, isoenzimas, adenosindesaminasa, líquido cefalorraquídeo.

\section{Abstract}

Introduction: Tuberculosis (TB) is a leading cause of death worldwide. Meningeal tuberculosis is a complication that requires early detection and immediate installation of appropriate therapy. Given the low sensitivity of sputum smear and culture of bacillus tuberculosis, other tools should be explored in the differential diagnosis of tuberculous meningitis such as adenosine deaminase (ADA) isoenzymes. Objectives: To determine ADA isoenzymes activity in cerebrospinal fluid (CSF) of patients with tuberculous meningitis. Design: Descriptive study, with non-probability sampling. Institution: Biochemistry and Nutrition Research Center, Universidad Nacional Mayor de San Marcos, Lima, Peru. Biological materials: Samples of CSF from patients with clinical symptoms of tuberculous meningitis. Interventions: Samples of CSF with ADA activity above $9 \mathrm{U} / \mathrm{L}$ were stored at $-40^{\circ} \mathrm{C}$ until vertical electrophoresis was run. Mann Whitney statistical analysis was used with 0.1 significance level to compare meningeal tuberculosis ADA values with other nervous system diseases. Main outcome measures: Isoenzymes ADA1m, ADA1cp, and ADA2. Results: Median total ADA enzyme activity in CSF in all patients with TB meningitis was higher than in other parainfective diseases of the central nervous system. In both cases ADA1cp had greater contribution to total ADA and showed larger increase in tuberculous meningitis. ADA2 increase was higher in meningeal tuberculosis compared with other CNS diseases. Conclusions: CSF electrophoresis distinguishes ADA isoenzymes, showing elevated levels of ADA2 in meningeal tuberculosis as a result of increased monocyte-macrophage cell line.

Key words: Tuberculosis/meningeal, isoenzymes, adenosine deaminase, cerebrospinal fluid.

\section{An Fac med. 2012;73(3):199-203}

\section{INTRODUCCIÓN}

La meningitis tuberculosa (MT) es la inflamación de las leptomeninges por Mycobacterium tuberculosis y la forma más grave de tuberculosis (TB) extrapulmonar. La inespecificidad de sus síntomas, la lentitud en el diagnóstico etiológico y las secuelas neurológicas que puede producir, hacen de esta enfermedad un problema importante de salud pública, tanto en países desarrollados como en países en desarrollo ${ }^{(1)}$.

La TB es una enfermedad infecciosa prevenible y curable que se transmite por el aire. En el 2008, 9,4 millones de personas contrajeron la enfermedad y 1,8 millones fallecieron ${ }^{(2)}$. El número de enfermos ha aumentado en muchos países en donde los programas de control no han resultado muy satisfactorios, incrementándose aún más en las poblaciones de riesgo (pobreza, inmigrantes) ${ }^{(3)}$.

El 86\% de todos los casos de TB en Lima Metropolitana son notificados en 18 de sus 43 distritos; $83 \%$ de los 
distritos de este grupo comunican casos de TB MDR por encima del promedio de Lima Metropolitana ${ }^{(4)}$. En el 2002, en el Hospital Dos de Mayo de Lima, del grupo de pacientes que presentó tuberculosis (89 casos), 16 pacientes manifestaron antecedente de enfermedad previa, de los cuales 56,3\% (9 casos) tuvo tuberculosis pulmonar, $25 \%$ (4 casos) tuberculosis miliar, 12,5\% (2 casos) tuberculosis meníngea y hubo un caso de tuberculosis enteroperitoneal (6,3\%); $17,9 \%$ (16 casos) tuvo contacto intradomiciliario y $5,6 \%$ (5 casos) contacto extradomiciliario. Cabe resaltar que estas cifras podrían ser mayores, ya que la mayoría de los pacientes puede ignorar el estado de salud de las personas con las que convive y/o trabaja ${ }^{(5)}$.

La medida preventiva más eficaz para evitar el contagio es eliminar las fuentes de infección presentes en la comunidad por medio de la detección, diagnóstico precoz y tratamiento oportuno y completo de los casos de tuberculosis ${ }^{(6)}$.

La TB extrapulmonar puede afectar cualquier órgano y destruirlo si no es tratada a tiempo; además, la condición es una gran simuladora, que aparece bajo la forma de otras enfermedades ${ }^{(7)}$. Este tipo de tuberculosis representa el 15\% de las presentaciones de la enfermedad, incluyendo la MT, que está presente en $4 \%$ de todos los casos; a pesar del tratamiento adecuado, la mortalidad llega a alcanzar cifras entre 15 y 40\%. La MT no solo compromete las meninges basales, sino también el parénquima cerebral, las arterias y venas cerebrales ${ }^{(8)}$. La MT representa la cuarta complicación neurológica más importante y la segunda enfermedad oportunista más frecuente después de la toxoplasmosis cerebral, en Europa, debido en gran parte a la alta prevalencia de pacientes infectados por VIH en países donde la tuberculosis es endémica ${ }^{(9,10)}$.

La MT requiere detección temprana e instalación de la terapia apropiada. El diagnóstico y tratamiento temprano pueden llevar a una recuperación en el $100 \%$ de los casos, sin secuela alguna.
La enfermedad no tratada es mortal en 3 a 8 semanas desde su presentación y conlleva a un riesgo de daños neurológicos severos si la terapia tarda. Usualmente, el foco de infección es pulmonar y la bacteria alcanza el sistema nervioso central por diseminación hematógena o en algunos casos por extensión local; estudios retrospectivos confirman que al menos 75\% de los pacientes presentó infección dentro de los 12 meses antes de su admisión por meningitis ${ }^{(11)}$.

La sensibilidad baja de la baciloscopia en LCR (22\%) y el prolongado periodo del cultivo ( 2 a 8 semanas) no son útiles en la conducta terapéutica inicial; todo esto ha hecho que la prueba de adenosina deaminasa (ADA) sea frecuentemente utilizada en nuestro medio para confirmar la sospecha clínica de infección por Mycobacterium tuberculosis ${ }^{(12,13)}$.

Se ha encontrado niveles elevados de ADA en otras enfermedades del sistema nervioso central, incluyendo la criptococosis meníngea, toxoplasmosis cerebral, meningitis bacteriana e incluso enfermedades no infecciosas, como hemorragia subaracnoidea ${ }^{(14)}$, y en otro tipo de enfermedades, tales como tifoidea, mononucleosis infecciosa y ciertas neoplasias, especialmente las de origen hematopoyético ${ }^{(15,16)}$. Para el caso de meningitis tuberculosa, la prueba de ADA ha demostrado tener una sensibilidad de $66 \%$ y hasta $100 \%$, con especificidad de 87 a $99 \%{ }^{(17)}$.

La adenosina deaminasa participa en la vía degradativa de adenosina monofostato, nucleótido que tiene rol central en el desarrollo del sistema inmune. Cataliza la desaminación de adenosina y desoxiadenosina en inosina y desoxiinosina, respectivamente, y es expresada prácticamente en todas las células y tejidos de los mamíferos; su actividad puede variar de acuerdo al tejido y las especies ${ }^{(18,19)}$. Se define una unidad de adenosina deaminasa como la cantidad de enzima requerida para producir 1 $\mu$ mol de amonio por minuto a partir de adenosina, en condiciones de ensayo estándar ${ }^{(20)}$. En 1973, Piras y col. seña- laron que había elevación de ADA en MT con relación a formas de meningitis virales. Para el caso de tuberculosis meníngea se ha propuesto valores de corte de 9 U/L y se ha aceptado además que el valor normal en LCR debe de estar por debajo de $6 \mathrm{U} / \mathrm{L}^{(21)}$.

Se distinguen 3 formas moleculares de la enzima: ADA1, proteína monomérica de $35 \mathrm{KDa}$; ADA 1cp (compuesto por dos moléculas de ADA1 unidas por una proteína recombinante) con $280 \mathrm{KDa}$; y ADA2. Dos isoformas de adenosina deaminasa, ADA1 y ADA2, actúan sobre dos sustratos diferentes, ADA 1 sobre adenosina y ADA2 sobre 2-deoxioadenosina, ensayo que se usa para distinguirlos ${ }^{(22,23)}$. Las isoenzimas ADA1, ADA1cp, y ADA2 tienen propiedades bioquímicas únicas; así, ADA1 está presente en todas las células, con alta actividad en linfocitos y monocitos, mientras que ADA2 aparece solo en monocitos ${ }^{(24)}$.

Debido al incremento de casos de tuberculosis en nuestro medio, y por los problemas asociados al diagnóstico oportuno, nos planteamos realizar el estudio de isoenzimas en LCR de pacientes del Hospital Nacional Guillermo Almenara Irigoyen (HNGAI) con TB; luego, cuantificar las actividades de estas isoenzimas para determinar su contribución en tuberculosis meníngea; y proponer la determinación de estas isoenzimas como herramienta de ayuda para el diagnóstico diferencial.

\section{MÉTODOS}

Las muestras de LCR fueron obtenidas del laboratorio de bioquímica del HN GAI. Para la preparación de los estándares se usó un pool de glóbulos rojos para isoenzima ADA1, pool de sueros normales para proteínas recombinante y suero de pacientes con hepatitis crónica para ADA2.

Se revisó las historias clínicas de pacientes que presentaron valores de ADA en LCR mayores a $9 \mathrm{U} / \mathrm{L}$; se tomó en cuenta además los parámetros 


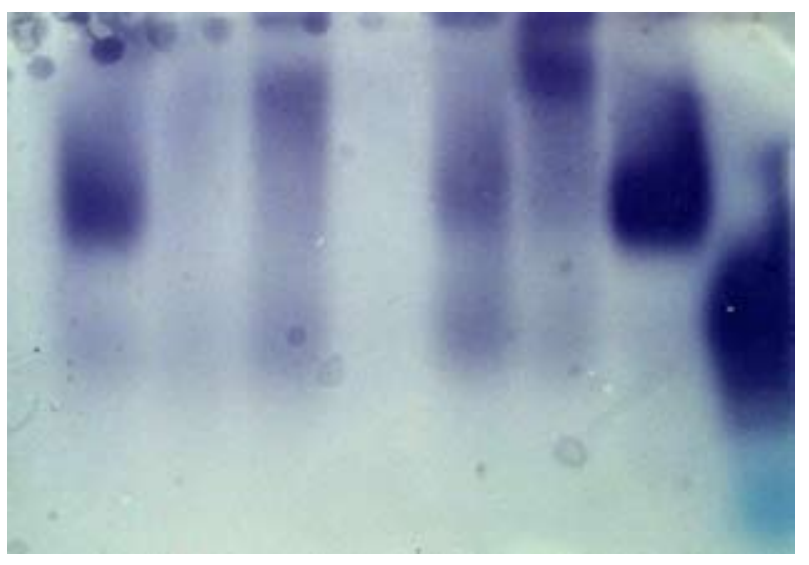

Figura 1. Representación esquemática de las tres formas moleculares de ADA. Separación de las isoenzimas de ADA en líquido cefalorraquídeo por electroforesis en gel de poliacrilamida.

bioquímicos de glucosa y proteínas, así como la celularidad en cada uno de los casos.

Se preparó los estándares de adenosina deaminasa ADA1 siguiendo la técnica descrita por Buel y MacQuarrie ${ }^{(25)}$; para ADA1cp se usó la técnica descrita por Ungerer y col. ${ }^{(26)}$, y para el estándar de ADA2 se tomó en cuenta el hallazgo de Fumie Kobayashi ${ }^{(22)}$.

Las isoenzimas de ADA presentes en LCR fueron separadas usando la técnica Laemli (27) para electroforesis no denaturante en geles de poliacrilamida al 5\%, la misma que fue realizada en una cámara vertical de dimensiones de 80 x 90 x 1,5 mm. Se hizo una preelectroforesis por 20 minutos a $20 \mathrm{~mA} \mathrm{y}$ a $4^{\circ} \mathrm{C}$, luego de la cual se aplicó $120 \mu \mathrm{L}$ de cada unas de las muestras de LCR y $30 \mu \mathrm{L}$ de los estándares. Finalmente, la corrida electroforética fue realizada a $30 \mathrm{~mA}$ durante 5 horas, a $4^{\circ} \mathrm{C}$ en buffer fosfato $0,1 \mathrm{M}$, a pH 6,7.

Como las isoenzimas tienen cada una diferentes cargas a $\mathrm{pH} 6,7$, emigran en tres distintas regiones del electroforegrama, por lo que pueden ser localizadas por medio de su capacidad para catalizar la reducción de un colorante o sustrato a su forma colorida.

La determinación de la actividad de cada isoenzima se efectuó en un buffer de coloración compuesto de $0,3 \mathrm{~mol} / \mathrm{L}$ de tris- $\mathrm{HCl}$ y $0,2 \mathrm{~mol} / \mathrm{L}$ de histidina a $\mathrm{pH} 7,8$. Para la coloración enzimática se usó la siguiente mezcla: $6 \mathrm{mg}$ de adenosina, $1,5 \mu \mathrm{L}$ de nucleósido fosforilasa, 3,25 $\mu \mathrm{L}$ de xantina oxidasa, 3 mg de MTT (3-[4,5-dimetiltiazol-2-yl] -2,5-difenil-tetrazolium bromuro), 0,20 mg de fenazina metasulfato (PMS) y 4 $\mathrm{mg}$ de fosfato de sodio, todo esto en 3 $\mathrm{mL}$ de buffer de coloración previamente preparado.

Terminada la corrida electroforética, se retiró el gel y se colocó sobre una lámina de vidrio al que se le roció buffer fosfato; aparte, se tomó una membrana de acetato de celulosa y se sumergió en la mezcla de coloración enzimática recién preparada, para humedecerla completamente; luego, se llevó esta membrana sobre el gel de poliacrilamida y se incubó a $37^{\circ} \mathrm{C}$ durante 1 hora; las isoenzimas fueron visualizadas exclusivamente en las membranas de acetato de celulosa. La actividad relativa de cada isoenzima fue determinada por densitometría de geles usando EZC SCANN de Helena Laboratories. Como control interno de calidad, todos los procedimientos fueron realizados por duplicado y se corrió estándares en cada uno de los geles. La posición relativa de cada isoenzima fue determinada siguiendo el patrón mostrado por los estándares, tal como se muestra en la figura 1.
Para el análisis de dato, se empleó estadística no paramétrica usando la prueba de Mann Whitney, con un nivel de significancia de 0,10 entre las medianas de los valores de las isoenzimas de ADA.

\section{RESULTADOS}

De las 67 historias clínicas revisadas en el periodo setiembre 1998 hasta enero 2000 , de pacientes con valores de adenosina deaminasa mayores de $9 \mathrm{U} / \mathrm{L}$, se encontró que 22 casos correspondían a MT.

En el análisis comparativo de los casos de MT versus otras enfermedades del SNC, se encontró que los valores medios de adenosina deaminasa total eran 22,5 y 13,5 U/L (tabla 1). Las medianas de la actividad enzimática de ADA total en LCR de pacientes con MT fueron mayores que en otras enfermedades parainfectivas del sistema nervioso central. La contribución de cada isoenzima a la actividad de ADA total fue como sigue: isoenzima ADA1m muy poca contribución, tanto en MT como en otras patologías; ADA1cp tuvo mayor contribución para ADA total en ambos casos, hallándose incrementado en MT; en tanto que, el incremento fue mayor para el caso de ADA2 en MT comparada con otras enfermedades del SNC (tabla 2).

\section{DISCUSIÓN}

A partir del estudio de Barbieri ${ }^{(28)}$, se utiliza la prueba (test) de ADA como herramienta de ayuda diagnóstica en MT, en la que se indica un valor de corte de 9 U/L. Sin embargo, se conoce que en otras enfermedades del sistema nervioso central existen niveles considerablemente altos de esta enzima en el LCR, tal como lo indica Chawla ${ }^{(12)}$. Por lo que el presente estudio estuvo enfocado en discriminar las isoenzimas de ADA para lograr su utilidad diagnóstica diferencial ${ }^{(28)}$, demostrándose la presencia de tres isoenzimas de ADA a nivel de LCR: una que se localiza en el 
Tabla 1. Valores medios de adenosina deaminasa en líquido cefalorraquídeo en meningitis tuberculosa y otras patologías del SNC.

\begin{tabular}{cccccc} 
& \multicolumn{2}{c}{ Meningitis tuberculosa } & & \multicolumn{2}{c}{ Otras patologías } \\
\cline { 2 - 3 } \cline { 5 - 6 } Actividad ADA & Media & Rango & & Media & Rango \\
\hline
\end{tabular}

Tabla 2. Porcentaje de contribución de cada isoenzima a nivel de adenosina deaminasa total por patologías.

\begin{tabular}{cccccc} 
Porcentaje de & \multicolumn{2}{c}{ TB meníngea } & & \multicolumn{2}{c}{ Otras patologías } \\
\cline { 2 - 3 } \cline { 5 - 6 } contribución & Media & Rango & & Media & Rango \\
\% ADA1m & 6,78 & $0-22,1$ & & 11,38 & $0-32,6$ \\
\% ADA1cp & 50,4 & $17,8-89,3$ & & 60,79 & $24,6-100$ \\
\% ADA 2 & 42,71 & $10-72,5$ & & 27,83 & $0-59,7$ \\
\hline
\end{tabular}

punto de aplicación u origen de la corrida electroforética, que corresponde a isoenzima ADA2; otra en posición intermedia ADA1cp; y la última posición aniónica que corresponde a ADA1m (figura 2).

En el presente estudio se ha encontrado que los patrones electroforéticos de las isoenzimas de ADA en geles unidimensionales no denaturantes, a partir de muestras de LCR con valor de corte de $9 \mathrm{U} / \mathrm{L}$, presentan una variación significativa en cada nivel de isoenzimas, patrones que guardan similitud con los encontrados por Gakis ${ }^{(24)}$, Ungerer ${ }^{(26)}$ y Kobayashi ${ }^{(25)}$, con la diferencia que estos estudios no fueron realizados con LCR sino mas bien con otros líquidos y efusiones corporales. El incremento de ADA en LCR de pacientes con MT es debido al predominio celular mononuclear, ya que es conocido que los linfocitos y monocitos tienen mayor actividad ADA que otros tipos celulares y tejidos ${ }^{(24)}$.

En MT, la mayor contribución a la actividad de adenosina deaminasa total es debida a ADA1m producida por linfocitos y monocitos y ADA2 producida por monocitos ${ }^{(24)}$. En contraste, en el presente estudio se encontró escasa o ninguna actividad de ADA1m y mayor actividad de ADA1cp. La proteína cp, que da origen a ADA $1 \mathrm{cp}$, probablemente viene del plasma, ya sea por un daño de la barrera hematoencefálica o como consecuencia del proceso inflamatorio, según refieren Chawla y Gakis ${ }^{(12,24)}$. Sea cual fuere su origen en el LCR, esta proteína $\mathrm{cp}$ se encuentra en concentraciones altas en el plasma y al pasar al LCR permite la formación de más ADA1cp a expensas de ADA1m ${ }^{(26)}$. La presencia de proteína cp en LCR incrementado por efecto del daño en la barrera hematoencefálica es evidenciada por la hiperproteinorraquia marcada presente en LCR de pacientes con MT. Cabe indicar que la isoenzima ADA1cp también se encuentra incrementada en otras enfermedades del sistema nervioso central, por lo que para una correcta interpretación se debe de tomar en cuenta los valores de ADA2. Usando el estadístico de Mann Whitney, se encontró que las medianas de ADA total en LCR de pacientes con tuberculosis meníngea fueron mayores que en otras enfermedades parainfectivas del sistema nervioso central. La misma prueba demostró que los valores de ADA2 presentaron incremento en MT respecto a otras enfermedades del sistema nervioso, con significancia estadística. Con lo que se concluye que esta prueba puede ser usada como ayuda para el diagnóstico diferencial de MT.

\section{AGRADECIMIENTOS}

A la Dra. María Raquel Oré Sifuentes por habernos brindado las facilidades técnicas de los Laboratorios de Química Bioorgánica del Centro de

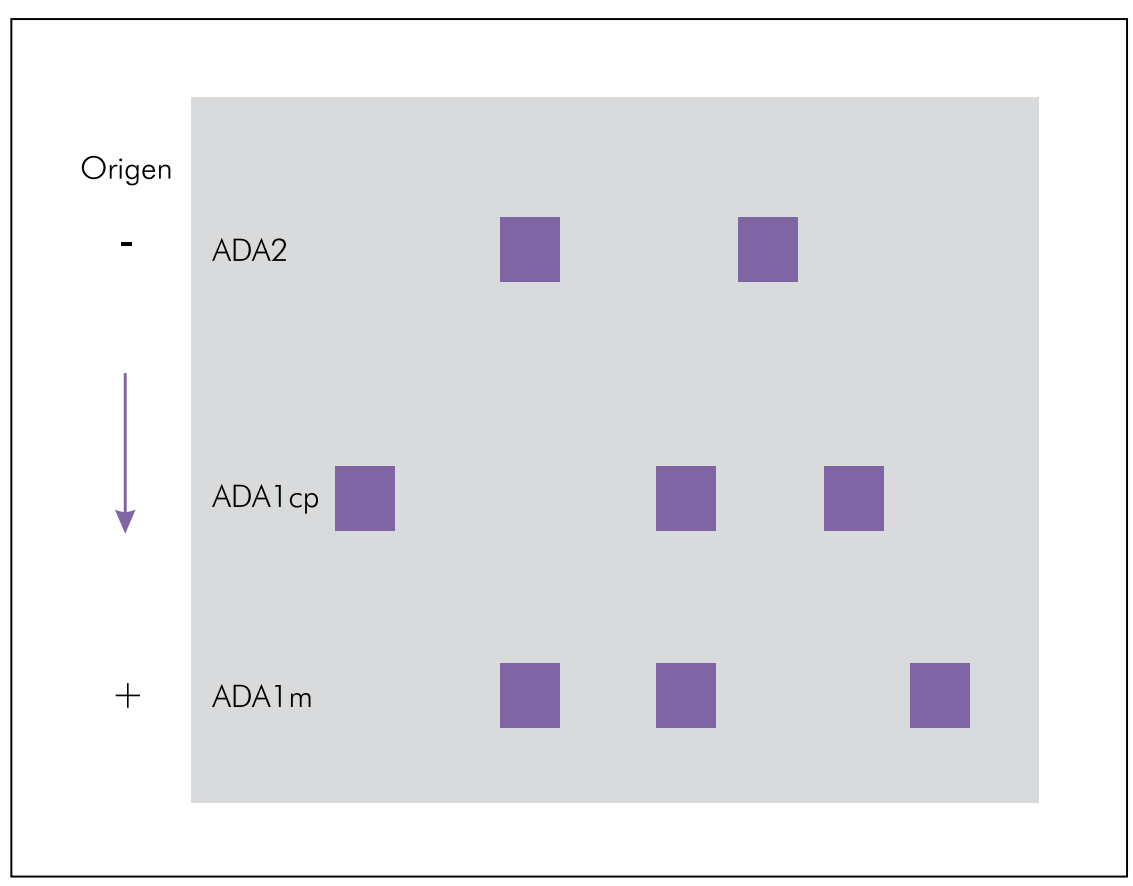

Figura 2. Muestra la posición relativa de cada isoenzima: ADA 2 en posición catódica (al inicio de la corrida), ADA1cp en posición intermedia y ADA $1 \mathrm{~m}$ en posición anódica. 
Investigación de Bioquímica y Nutrición, sin lo cual no hubiese sido posible llevar a cabo la presente investigación.

Al Lic. TM Miguel Sandoval Vegas por la revisión del manuscrito original y por valiosas sugerencias para la edición final.

\section{REFERENCIAS BIBLIOGRÁFICAS}

1. Enberg M, Quezada M, De Toro C, Fuenzalida L. Meningitis tuberculosa en adultos: análisis de 53 casos. Rev Chil Infect. 2006;23(2):134-9.

2. Control mundial de la tuberculosis - Informe OMS 2011.

3. Farga V. Tuberculosis, lo que hay que saber. Rev Chil Enf Respir. 2008;24:317-22.

4. Bonilla C. Situación de la tuberculosis en el Perú. Acta Med Per. 2008;25(3):163-70.

5. Corilloclla R. Tuberculosis pulmonar: enfermedad marcadora de sida en pacientes $\mathrm{VIH}$ positivos en el Hospital Nacional Dos De Mayo. 2004 - 2006. Tesis para optar el titulo de Medico Cirujano General. Lima, 2008.

6. Norma Técnica de Salud para el Control de la Tuberculosis. Perú. NTS N041/MINSA/DGSPV.01, 2006.

7. Arciniegas W, Orjuela D. Tuberculosis extrapulmonar. Biomédica. 2006;26:71-80.

8. Vila B, Martín C, Leal P, Rodriguez ML. Meningitis tuberculosa. A propósito de un caso. Med Intensiva. 2008;32(5):258-61.

9. Mak W, Cheung R, Ho S, Tsang K, Fong G. Tuberculous meningitis in Hong Kong: experience in a regional hospital. Int J Tuberc Lung. 1998;2:1040-3.

10. Úriz J, Repáraz J, Castiello J, Sola J. Tuberculosis en pacientes infectados por el VIH. An Sist Sanit Navar. 2007;30(Supl 2):131-42.
11. Farreras P, Rozman C. Medicina Interna. Decimosexta edición. España: Editorial Elsevier. 2008.

12. Chawla R, Seth R, Raj B, Saini A. Adenosine deaminase levels in cerebrospinal fluid in tuberculosis and bacterial meningitis. Tubercle. 1991;72:190-2.

13. Egido J, Gonzales J, Cuba E. False positive of ADA determination in CSF. Acta Neurol (Napoli). 1994;16:288-90.

14. Ena J, Crespo J, Valls V. Adenosine deaminase activity in cerebrospinal fluid a useful test for meningeal tuberculosis in patients with AIDS. J Infect Dis. 1988;158:4.

15. Saravia de Cunha, Gaspar E, Melico-Silvestre A. Neurobrucellosis - another cause of increased adenosine deaminase activity in cerebrospinal fluid. J Infect Dis. 1990;161:156-7.

16. Carstens M, Burgess L, Maritz F, Talijaard J. Isoenzymes of adenosine deaminase in pleural effusions: a diagnostic tool? Int J Tuberc Lung Dis. 1998;2:831-5.

17. Mejía A, Pajares V, Fernández A. Adenosina deaminasa sérica como marcador de la respuesta terapéutica en TBC pulmonar. Diagnostic. 1998;37:328-34

18. Ribera E, Martinez J, Ocana I, Ruiz I, Jimenez J, Encabo G, Segura R, Pascual C. Diagnostic value of ascites gamma interferon levels in tuberculous peritonitis. Comparison with adenosine deaminase activity. Tubercle. 1991;72:193-7.

19. Ungerer J, Oosthuizen H, Bissbort S, Vermaak W. Serum Adenosine deaminase: isoenzymes and diagnostic application. Clin Chem. 1992;38:1322-6.

20. Akedo H, Nishihara H, Shinkai K, Komatsu K, Ishikawa S. Multiple forms of human adenosine deaminase. Biochim Biophys Acta. 1972;276:257-71.

21. Conde Y. Células en el líquido pleural. Su valor en el diagnóstico diferencial de los derrames pleurales. Rev Clin Esp. 1994;73.

22. Kobayashi F, Ikeda T, Marumo F, Sato Ch. Adenosine deaminase isoenzymes in liver disease. Am J Gastroent. 1993;2:266-71.
23. Van Der Weyden M, Kelley W. Human adenosine deaminase. Distribution and properties. J Biol Chem. 1976;251:5448-56.

24. Gakis C. Adenosine deaminase levels in cerebrospinal fluid in tuberculosis and bacterial meningitis. Tubercle Lung Dis. 1995;76:372-3.

25. Buel E. Mac Quarrie. Purification of adenosine deaminase from human red blood cell. Preparative Biochemistry. 1981;11:363-80.

26. Ungerer J, Oosthuizen H, Retief J, Bissbort S. Significance of adenosine deaminase activity and isoenzymes in tuberculous effusions. Chest. 1994;106:33-7.

27. Andrews AT. Electrophoresis theory. Techniques and biochemical and clinical applications. Second Edition. Oxford: Edit Clarendon Press. 1986:24.

28. Barbieri R. Determinación de Actividad de Adenosina Deaminasa en Líquidos Biológicos, para el diagnóstico de la Tuberculosis Pulmonar y Extrapulmonar. Hospital Nacional Edgardo Rebagliati Martins. Tesis para optar el título de Magister en Patología Clínica. Lima- Perú: UNMSM. 1994.

\section{Financiamiento:}

Recursos propios y proyecto Fedu.

Conflicto de intereses:

Los autores indican que no hubo conflictos de interés en el presente trabajo.

\section{Autor corresponsal:}

Heli Jaime Barrón Pastor

helibarron@unmsm.edu.pe

Centro de Investigación de Bioquímica y Nutrición, Facultad de Medicina, Universidad Nacional Mayor de San Marcos

Av. Grau 755, Lima 1, Perú

Teléfono celular: 990229858 